\title{
LARYNGEAL MASK VENTILATION DURING TRACHEOSTOMY IMPROVES INTRAOPERATIVE HEMODYNAMIC STABILITY IN PATIENTS UNDERGOING TOTAL LARYNGECTOMY
}

\author{
Tz. Marinov', T. M. Popov², M. Belitova ${ }^{1}$ \\ 1Department of Anesthesiology and Intensive Care, Medical University - Sofia, Bulgaria \\ ${ }^{2}$ Department of ENT, Medical University - Sofia, Bulgaria
}

\begin{abstract}
Background and objectives: Laryngectomy with extensive extirpational neck dissection is still the treatment of choice for patients with advanced laryngeal cancer. During the initial part of laryngectomy - tracheostomy, there is a significant upper airway obstruction, caused by the cancer process itself and worsened by surgical pressure and manipulation during creation of tracheostomy. This study aims to make comparative assessment of the patient's hemodynamic parameters, operated using three of the most popular approaches during tracheostomy: local anesthesia with preserved spontaneous ventilation; general anesthesia with ventilation by endotracheal intubation and general anesthesia with ventilation by laryngeal mask airway. Methods: A prospective cohort study was conducted in a tertiary referral center. Sixty patients with advanced laryngeal cancer appointed for total laryngectomy, were enrolled in the study. They were randomly assigned into three groups, according to the ventilation method used during the tracheostomy. Results: Patients who underwent tracheostomy under local anesthesia displayed statistically the highest levels of SAP, DAP, MAP and heart rate intraoperatively. The group of patients who underwent tracheostomy with endotracheal intubation, also displayed significantly higher levels of hemodynamic parameters during the procedure compared with the group with laryngeal mask airway ventilation, despite the fact that both groups were under general anesthesia. Conclusions: To our knowledge, this is the first study to demonstrate that laryngeal mask ventilation during tracheostomy improves intraoperative hemodynamic stability in patients undergoing total laryngectomy compared to endotracheal intubation.
\end{abstract}

Key words: tracheostomy, laryngectomy, laryngeal masks, hemodynamics, upper airway instrumentation, ventilation

Corresponding author: Assoc. Prof. Maya Belitova, MD, PhD, Department of Anesthesiology and Intensive Care, Medical University of Sofia, 1 "Sv.Georgi Sofijski" Blvd, 1431 Sofia, Bulgaria, Tel.: +359898 5514 65, e-mail: belitova@mail.bg

\section{INTRODUCTION}

T he human larynx plays a pivotal role in airway protection, respiration and phonation and laryngeal cancer is one of the most popular causes of laryngeal obstruction which compromises these vital functions. Laryngectomy with the appropriate type of neck dissection is still the golden standard treatment option for patients with advanced laryngeal cancer despite the rise of laryngeal preservation protocols during the last two decades [1]. The initial part of this operative procedure requires ensuring a tra- 
cheostomy, which would allow undisrupted surgical access to the upper airways without interfering with patient's ventilation. Creation of tracheostomy is one of the most challenging phases for the anesthesiologists during total laryngectomy, because of the need to ensure an anesthetic state, in face of unsecured airways, which are compromised from the tumor process causing different degrees of laryngeal obstruction. Also, since most of the patients indicated for this type of surgery suffer from severe comorbidities (e.g. cardiovascular disease, COPD, etc.) [2], intraoperative ventilation and hemodynamic stability are essential for the untroublesome course of this major operative procedure. In this study we make a comparative assessment of patients' hemodynamic parameters during tracheostomy, operated using three approaches: local anesthesia with preserved spontaneous ventilation; general anesthesia and ventilation with endotracheal intubation; general anesthesia and ventilation with laryngeal mask airway device.

\section{MATERIAL AND METHODS}

A randomized controlled trial with level of evidence $1 \mathrm{~b}$ was conducted in a university hospital department during the period 2013-2016. Sixty patients with histopathologically verified carcinoma of the larynx in advanced stage (T3/T4) were enrolled in the study. Informed consent was solicited from every patient and the protocol of the study was approved by the university's ethics committee. A standardized history was obtained for each patient. Detailed description of the endoscopic/microscopic direct laryngoscopy findings were recorded along with the computer tomography examination results. All patients underwent primary total laryngectomy, none had a preoperative tracheostomy and none of them had decompensating dyspnea prior to surgery. According to the CottonMyer scale, we categorized the level of laryngeal obstruction in every patient by using preoperative video laryngoscopy. Patients were randomly assigned to the following groups:

- Group $1(\mathrm{n}=20)$ : tracheostomy was performed under local anesthesia (LA) with preserved spontaneous ventilation; patients received supplementary oxygen via nasal oxygen catheter.

- Group 2 ( $n=20)$ : tracheostomy was performed under general anesthesia with an endotracheal intubation (ETI) as a mode of upper airway instrumentation.

- Group $3(n=20)$ : tracheostomy was performed under general anesthesia with a laryngeal mask airway (LMA) as a mode of upper airway instrumentation.
General anesthesia in patients from the cohort (Gr.2 and Gr.3), was given according to a standard unified protocol. All patients were monitored and recorded for: peripheral capillary oxygen saturation (SpO2); systolic (SAP), diastolic (DAP) and mean arterial blood pressure (MAP); heart rate (HR); continuous electrocardiography (ECG). The above parameters were recorded at predetermined time intervals: T0: 5th minute after premedication; T1: 3rd minute after beginning the tracheostomy; T2: 5th minute after beginning the tracheostomy; T3: 10th minute after beginning the tracheostomy; T4: 3rd minute after intubation through the tracheostomy; T5: 10th minute after intubation through the tracheostomy; T6: 20th minute after intubation through the tracheostomy; T7: 3rd minute after extubation; T8: 5th minute after extubation; T9: 10th minute after extubation.

Statistical analysis of the results was performed using SPSS software version 21.0. One-way ANOVA test with Tukey post hoc test was used to compare groups. If the homogeneity of variance was violated (Levene test, $p<0.05$ ), the Games-Howell post hoc test was used. In cases when the assumption of normality was violated (Shapiro-Wilk test of normality indicated $p<0.05$ ) we used the nonparametric Kruskal-Wallis test.

\section{RESULTS}

The mean age of the whole pool of patients was 61 years. There were no statistically significant differences in age, ASA score, hypertension levels, COPD and ischemic heart disease prevalence between the three different groups. Additionally, we categorized the level of obstruction in every patient according to the Cotton-Myer scale by using preoperative video laryngoscopy. Again, no significant differences in the levels of obstruction were found between the three groups. All of the patients enrolled in the study showed no signs of decompensated inspiratory dyspnea prior to surgery.

At T0 (premedication stage) the patients from all three groups displayed identical levels of systolic, diastolic, mean arterial blood pressure and heart rate (Figures 1, 2, 3 and 4).

After the surgical procedure was initiated with the tracheostomy, distinct differences in the hemodynamics of the three groups could be clearly seen.

Patients who underwent tracheostomy under local anesthesia displayed statistically the highest levels of SAP, DAP, MAP and heart rate intraoperatively during the tracheostomy compared with the other two groups. Interestingly, the group of patients who 
underwent tracheostomy with endotracheal intubation, also displayed significantly higher levels of SAP, DAP, MAP and heart rate during the procedure in comparison to the group with laryngeal mask airway ventilation, despite the fact that both groups were under general anesthesia. This pattern of hemodynamic differences continued to be registered also at the beginning of the laryngectomy, at which point all patients were intubated with an endotracheal tube through the tracheostomy.

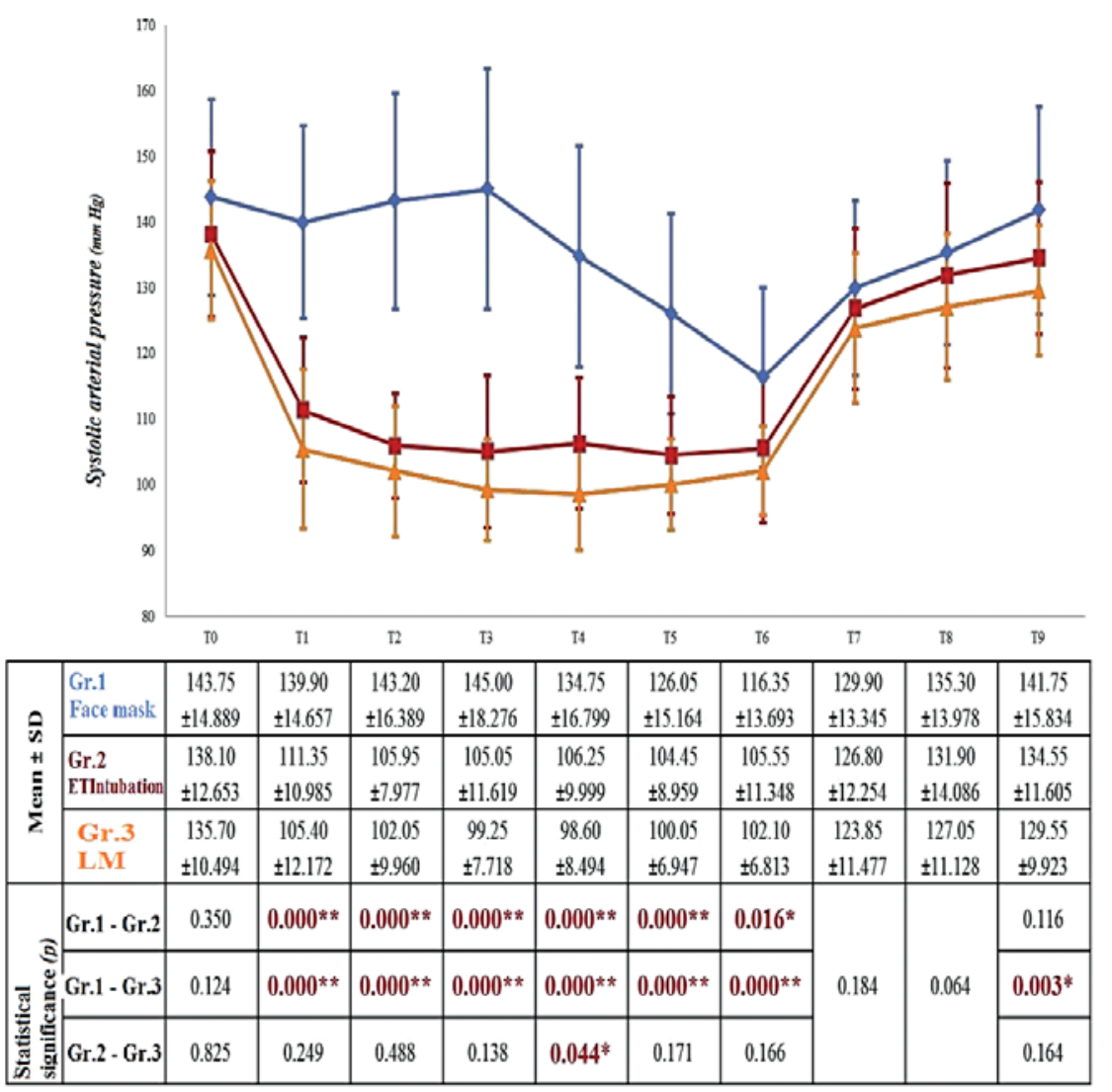

Fig. 1. Mean systolic arterial blood pressure levels for groups 1,2 and 3 at different surgical time points

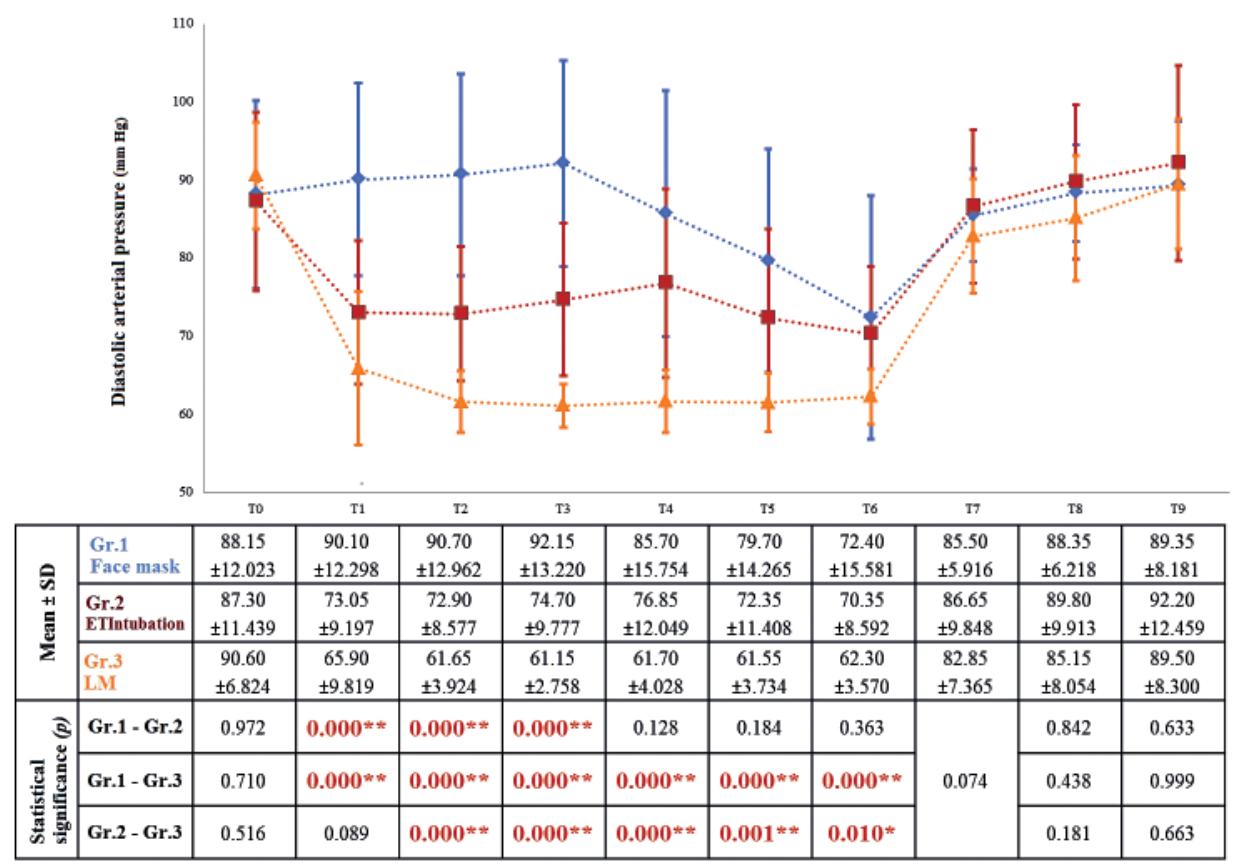

Fig. 2. Mean diastolic arterial blood pressure levels for groups 1,2 and 3 at different surgical time points 


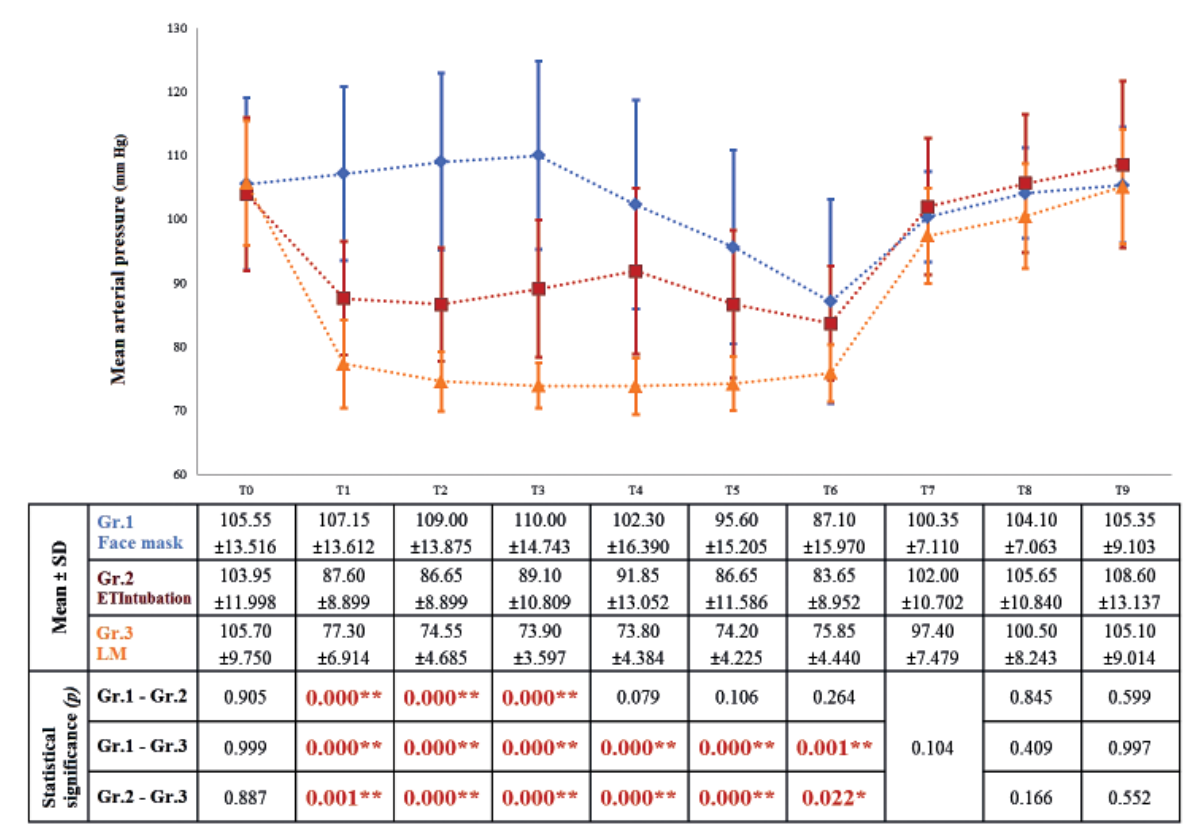

Fig. 3. Mean arterial blood pressure levels for groups 1,2 and 3 at different surgical time points

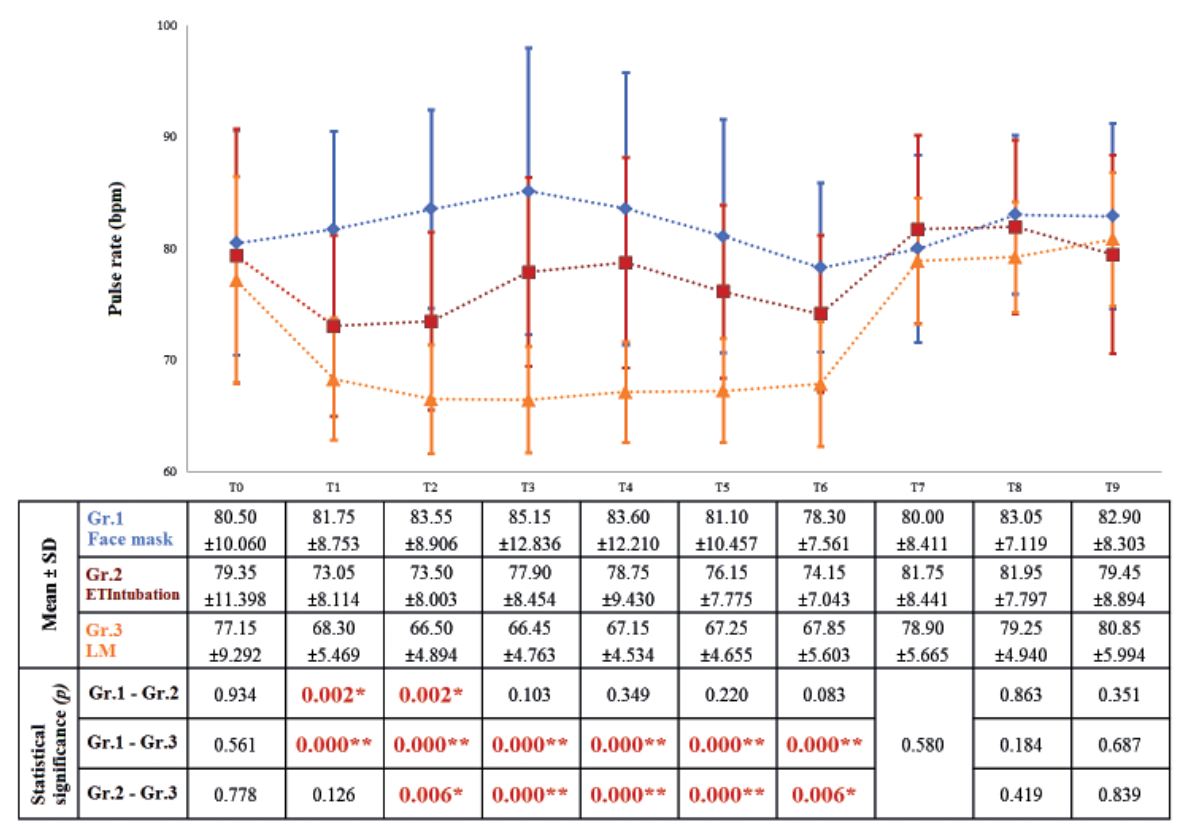

Fig. 4. Mean heart rate levels for groups 1,2 and 3 at different surgical time points

From the figures we could see significantly lower levels of all hemodynamic parameters (SAP, DAP, MAP and heart rate) obtained from patients in group 3 compared with the other two groups not only during the initial stages of operative procedure, but also during the first 20 minutes of the laryngectomy. We report no statistical difference in hemodynamic parameters between group 1 and 2 during the beginning of the laryngectomy, despite noting some tendencies. 20 minutes after the start of the laryngectomy, till the end of surgery, including the period after extubation, no differences in hemodynamic parameters were found between the three groups.

\section{DISCUSSION}

After a thorough literary investigation, we could not find a study designed similarly to the present one. Most studies focus on ventilation comparing laryngeal mask airway versus endotracheal intubation as airway device used during percutaneous tracheostomy, but none compares hemodynamic parameters [3, $4,5]$. Some studies assess the efficacy of laryngeal mask airway (LMA) ventilation in patients with airway stenosis [6] and report no major or minor complications. Our team also reported a cricotracheal resection in a case of subglottic stenosis with a lumen of 
$2.5 \mathrm{~mm}$ ventilated efficiently by using laryngeal mask airway [7]. Respectively, in the present study none of the cases with laryngeal mask airway suffered from any significant complications (including desaturations) despite considerable levels of obstruction caused by the tumor masses.

Group 1 showed the highest levels of SAP, DAP, MAP and heart rate, which could be easily explained by the adrenergic stress response during the local anesthesia [8]. One could see from the data that excessive catecholamine release during the tracheostomy has an impact even after induction of general anesthesia, i.e. the hemodynamic stability during the first part of the laryngectomy is directly influenced by the type of anesthesia for the tracheostomy. Even more intriguing is the impressive difference in all hemodynamic parameters between patients with endotracheal intubation and laryngeal mask airway. Even though both groups were operated under general anesthesia, intubated patients displayed firmly higher levels of blood pressure and pulse rates than those ventilated with laryngeal mask airway during tracheostomy and first 20 minutes of the laryngectomy. In comparison, hemodynamic parameters between group 1 and 2 at T4, T5 and T6 show only tendency, but no absolute statistical difference, which demonstrates the convincing advantages of laryngeal masks in these circumstances. We hypothesized that involvement of the laryngeal chemoreflex would explain the reported results. This reflex induces specific response initiated by receptors in the laryngeal lumen and among its numerous effects, a significant increase in blood pressure has been attributed to it $[9,10,11,12]$. Presumably, during intubation in patients with advanced laryngeal cancer, there is a significant mechanical compression on the endolarynx and respectively, on the tumor mass, because of the obstructed lumen, which could cause an induction of the laryngeal chemoreflex. An important part in the induction process could play the tumor microenvironment and more precisely the inflammatory component, abundant in biologically active molecules, given the reports demonstrating how a simple upper airway respiratory infection could significantly increase reactivity [13].

\section{CONCLUSION}

Anesthesia in head and neck surgery has always been demanding, as often pathology interferes with the anesthesiologist's field of work. To our knowledge, in this study we demonstrate for the first time in the scientific field that laryngeal mask ventilation during tracheostomy improves intraoperative hemodynamic stability in patients undergoing total laryngectomy compared to endotracheal intubation.
Conflict of interests: the authors declare no conflict of interests.

Acknowledgments: none.

\section{REFERENCES}

1. Dyckhoff G, Plinkert PK, Ramroth H. A change in the study evaluation paradigm reveals that larynx preservation compromises survival in T4 laryngeal cancer patients. BMC Cancer 2017; 17(1):609.

2. Rudolph E, Dyckhoff G, Becher $H$ et al. Effects of tumor stage, comorbidity and therapy on survival of laryngeal cancer patients: a systematic review and a meta-analysis. Eur Arch Otorhinolaryngol 2011; 268(2):165-79.

3. Price GC, McLellan S, Paterson RL, Hay A. A prospective randomized controlled trial of the LMA Supreme vs cuffed tracheal tube as the airway device during percutaneous tracheostomy. Anaesthesia 2014; 69(7):757-63.

4. Strametz R, Pachler C, Kramer JF et al. Laryngeal mask airway versus endotracheal tube for percutaneous dilatational tracheostomy in critically ill adult patients. Cochrane Database Syst Rev. 2014; 6:CD009901.

5. Pratt T, Bromilow J. Laryngeal mask airway for airway control during percutaneous dilatational tracheostomy. Anaesth Intensive Care 2011; 39(6):1009-13.

6. Pourciau DC, Hotard DP 3rd, Hayley S et al. Safety and efficacy of laryngeal mask airway ventilation in obese patients with airway stenosis. Laryngoscope 2017; doi: 10.1002/ lary.26684.

7. Popov TM, Marinov T, Rangachev J et al. Cricotracheal Resection in a Patient with Severe Subglottic Stenosis - Advantages of a Temporary Non-Cannulated Tracheostomy. Balkan Med J 2017; 34(2):165-167.

8. Middlehurst RJ, Gibbs A, Walton G. Cardiovascular risk: the safety of local anesthesia, vasoconstrictors, and sedation in heart disease. Anesth Prog 1999; 46(4):118-23.

9. Mutoh T, Tsubone H, Nishimura R, Sasaki N. Cardiovascular reflex mechanisms by topical instillation of capsaicin and distilled water into the larynx in anesthetized dogs. J Vet Med Sci 1997; 59(9):801-6.

10. Lee JC, Stoll BJ, Downing SE. Properties of the laryngeal chemoreflex in neonatal piglets. Am J Physiol 1977; 233(1):R30-6.

11. Nishino T. Physiological and pathophysiological implications of upper airway reflexes in humans. Jpn J Physiol 2000; 50(1):3-14. Review.

12. Wang $X$, Guo R, Zhao W. Distribution of Fos-Like Immunoreactivity, Catecholaminergic and Serotoninergic Neurons Activated by the Laryngeal Chemoreflex in the Medulla Oblongata of Rats. PLoS One 2015; 10(6):e0130822.

13. Nandwani N, Raphael JH, Langton JA. Effect of an upper respiratory tract infection on upper airway reactivity. $\mathrm{Br} \mathrm{J}$ Anaesth $1997 ; 78(4): 352-5$.

Received: March, 2019 - Accepted: April, 2019 